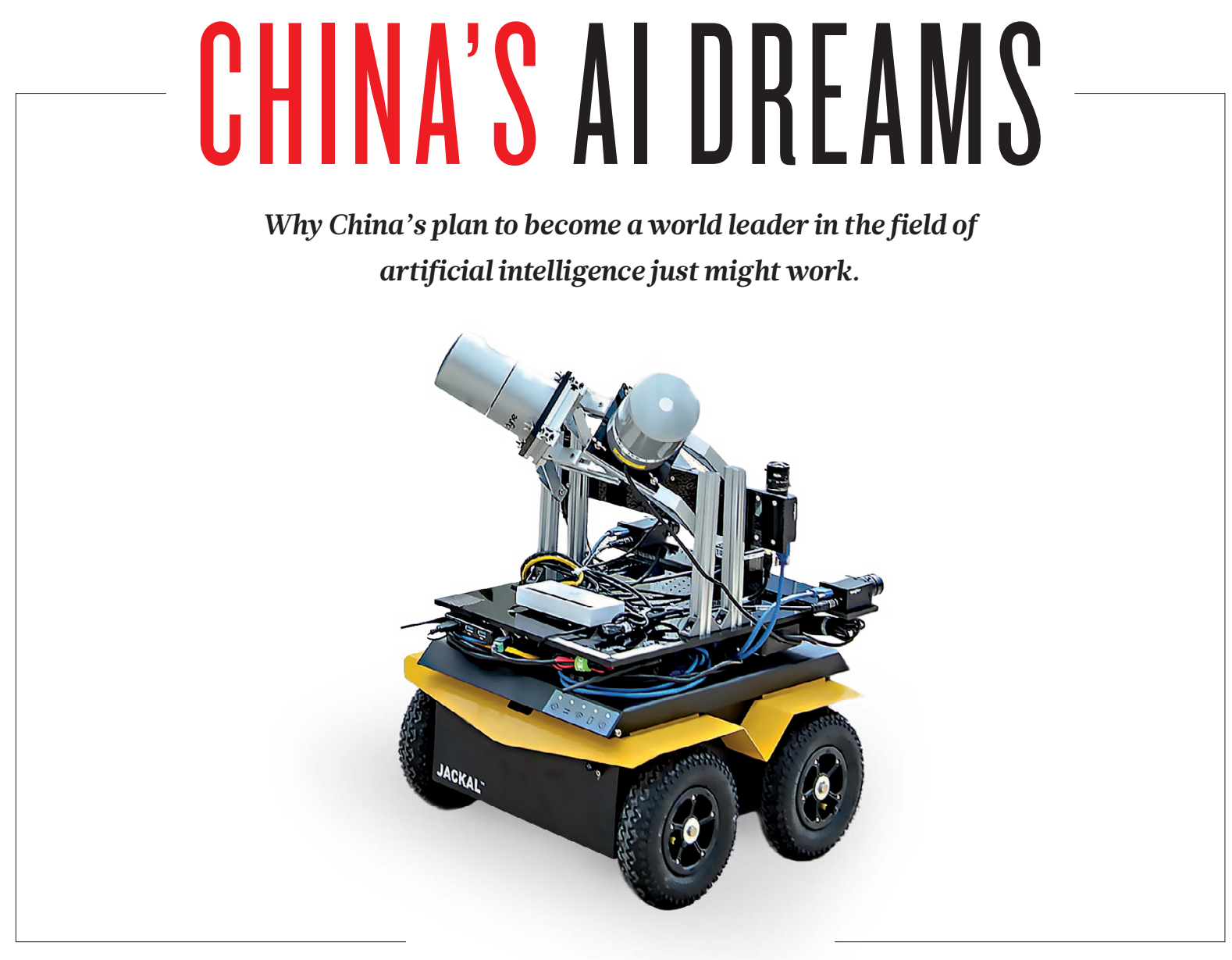

BY OWEN CHURCHILL

$\mathrm{L}$ ast year, China's chief governing body announced an ambitious scheme for the country to become a world leader in artificial intelligence (AI) technology by 2030. The Chinese State Council, chaired by Premier Li Keqiang, detailed a series of intended milestones in AI research and development in its 'New Generation Artificial Intelligence Development Plan', with the aim that Chinese AI will have applications in fields as varied as medicine, manufacturing and the military.

These AI ambitions, made public in July 2017, came as little surprise. 'Innovation' has been a favourite buzzword of China's leadership for several years, as the country seeks to transition from a production powerhouse to a centre of knowledge creation.

But China's AI aspirations are as economic as they are political, coming at a historic stall in the country's previously rapid growth — in 2016, the economy grew at its slowest rate since 1990. By 2020, the State Council's plan forecasts, the value of China's core AI industries should have exceeded 150 billion yuan (US $\$ 22.7$ billion), and the total for all related industries should be 1 trillion yuan. By 2030, it is hoped those figures will be 1 trillion yuan and 10 trillion yuan, respectively.

Much suggests that China is already on the right track. In 2014, the country overtook the United States in terms of the number of research publications it produced - and, crucially, the number of those that were cited - on the subject of deep learning; in the past two years, Chinese teams have dominated the prestigious ImageNet AI contest, in which researchers compete to see which algorithms can best recognize images; and Beijing-based technology giant Baidu, which in 2017 announced it was launching a deep learning research laboratory in collaboration with the Chinese government, says it will have self-driving vehicles powered by AI technology on Chinese public roads by 2020 .

But in its quest to become a leader in technology that does the job of people, China is uncharacteristically low on one thing: people. According to a recent LinkedIn report (see go.nature.com/2jvdcxe), there were more than 50,000 people in China's AI workforce in the first quarter of 2017. In the United States, a country with less than one-quarter of China's population, that number was above 850,000 . India is home to an AI workforce of more than 150,000.

"For the time being, talent remains a major bottleneck in China's advances in AI," says Elsa Kania, a Washington DC-based analyst specializing in China's emerging technologies and defence innovation. She says that it is a lack of experience as well as a lack of people that is afflicting the country's AI sector. Indeed, LinkedIn found that $38.7 \%$ of those working in China's AI sector have more than 10 years' experience, compared with $71.5 \%$ in the United States. That, she says, "will continue to necessitate active efforts to recruit foreign talent from Silicon Valley and elsewhere". In Beijing's tech hub Zhongguancun, steps have already been taken. In 2016, the local government made it easier for foreigners to gain permanent residency status, and in 2017 it introduced an advice service to support entrepreneurial newcomers with everything from Chinese company registration and taxation to finance and intellectual property rights.

Indeed, strengthening talent is considered a matter of "utmost 


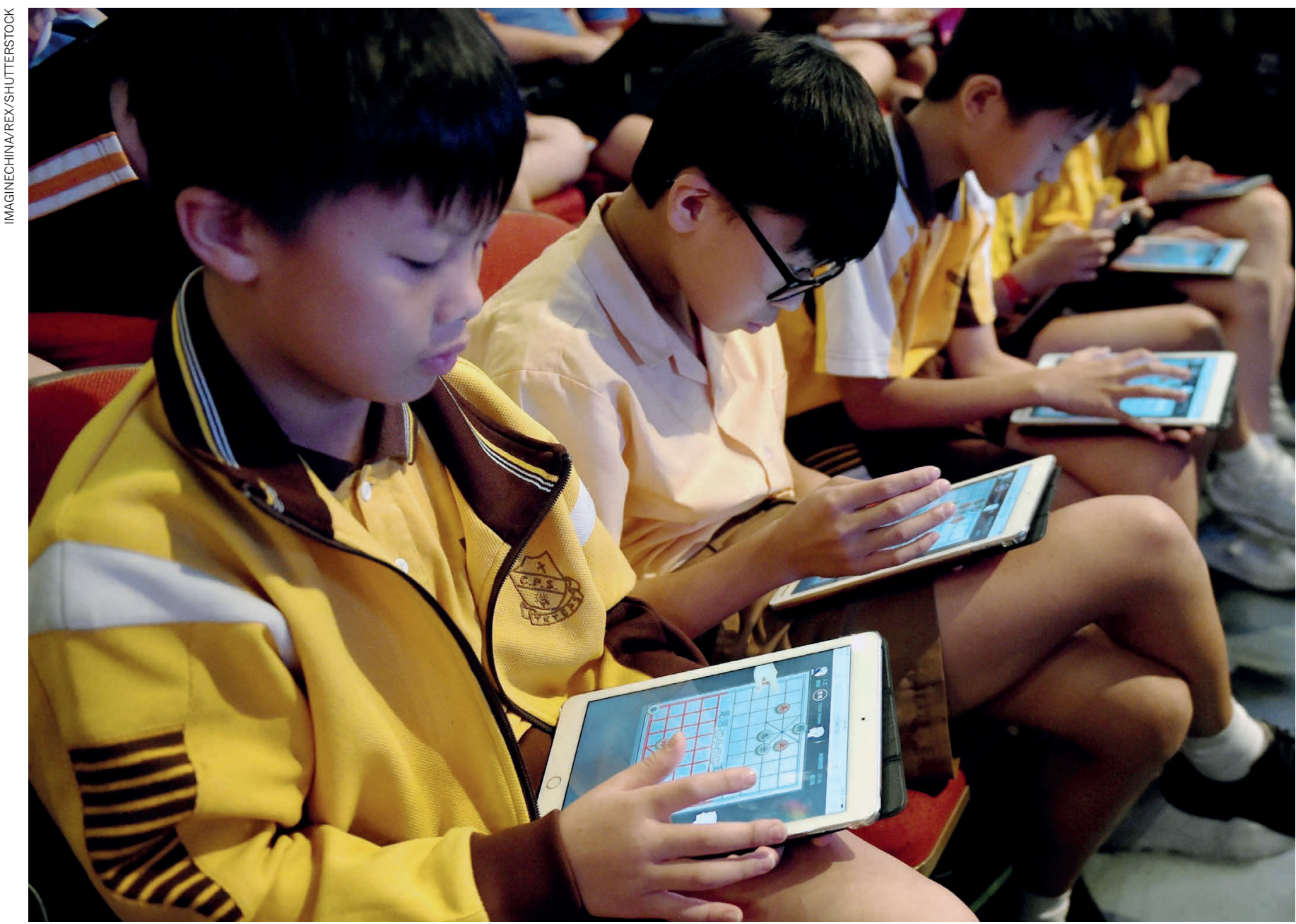

Students in Hong Kong play Chinese chess against an artificial intelligence system.

importance" in the government's AI development plan, which calls for "accelerating the introduction of first-rate global talent and young talent, to create a top talent base for China's AI". The plan makes specific reference to the Thousand Talents scheme, a government initiative that offers attractive financial packages to overseas scientists, both foreign and Chinese.

\section{DOLLARS AND DATA}

A number of factors will bolster efforts to make China an attractive place for top-tier AI talent, the first and foremost being the amount of public funding available to researchers. The value of this is particularly evident in today's world, says Kania, at a time when "the current US administration has not prioritized increased funding for research and development in AI".

Shu Chang, who works at the Shanghai-based AI start-up DeepBrain, says that "China's entire research environment might be better than that overseas." DeepBrain recently transitioned from producing intelligent hardware to focusing on cloud-based services. Referring to both AI and other technologies, Shu says that doctorate scholarships and research grants are easy to come by in China, especially when compared with Europe and the United States.

One of those benefiting from government capital is Sören Schwertfeger, a German robotics specialist who relocated to ShanghaiTech University to conduct research as an assistant professor. He says that he has received much more generous research funding from his university than he would have from institutions anywhere else in the world. Although specific purchases of research equipment must be signed off by the university, Schwertfeger says that his employer's input on the nature of his work is minimal. "I have my academic freedom and can do research on what I want.”

\section{"TALENT REMAINSA MAJOR BOTTLENECK IN CHINA'S ADVANCES."}

ShanghaiTech University was set up in 2013 by municipal authorities and the government-run Chinese Academy of Sciences, which said it was responding to "a nationwide call to put innovation at the heart of China's development". In short, says Schwertfeger, "Shanghai is spending lots of money". His department comprises a mixture of foreign staff and Chinese staff with international experience.

Alongside abundant capital is another resource crucial to any AI engineer: data. The most populous country in the world - home to 730 million Web users - is a massive, accessible, treasure trove of workable data that are generally available to companies. Although online data protection laws were tightened in 2017 to protect citizens' personal information, there are still legal means for companies to obtain data. 
- For example, Shu says that social media platform Weibo will provide developers with access to users' information, and such data are generally more available in China than abroad.

Schwertfeger says that his campus has around 3,000 cameras, a resource that could be tapped to drive facial-recognition research projects such as working out average class attendance. "Of course," he says, "not to spy on the students." Leaders in the AI field continue to cite ease of data access in China as a major selling point. As put by Wang Haifeng, vice president of Baidu, "data is the lifeblood of AI, and in China, we have excellent data pools".

\section{"WE ENCOURAGE OUR EMPLOYEES TO EXPRESS THEIR OPINIONS."}

It's an observation shared by others in the field. He Yong, an executive at DeepBrain, says that compared with citizens in Europe and the United States, the Chinese people have a much more relaxed attitude to personal privacy. He, whose company created the first Chinese-language mobile phone assistant - before Apple launched a Chinese version of Siri - says that AI companies in China "have an easier time both collecting data and putting it to use". DeepBrain's leadership is confident that such an environment makes China an attractive destination for global talent (see 'AI projects to watch') — although the company currently has no non-Chinese staff in its team of around 20. Nevertheless, the firm favours those with international experience, says Shu: "Technologies within AI, machine learning and deep learning all have their origins in the United States and Europe after all."

\section{LINKS TO THE UNITED STATES}

Taking a more proactive approach to enlisting foreign talent is Baidu, a company that has grown from a search engine into a multi-limbed tech giant increasingly focused on AI technology. The company's AI wing is actively seeking talent from abroad, where, says Wang, "the top echelon of AI talent is concentrated". As well as actively recruiting from US universities, Baidu operates two research and development centres in Silicon Valley, California, and opened another in Seattle, Washington, in October 2017. Such centres, says Wang, are crucial to not just harnessing international talent, but also ultimately channelling it towards China.

"Due to the United States' position as a hotbed of AI talent, our presence there is a main recruitment channel and a great access point," says Wang. The company's California-based AI research centre employs around 200 scientists - both Chinese and non-Chinese - contributing to the company's research in AI fields such as machine learning, big data, computer vision and natural language processing.

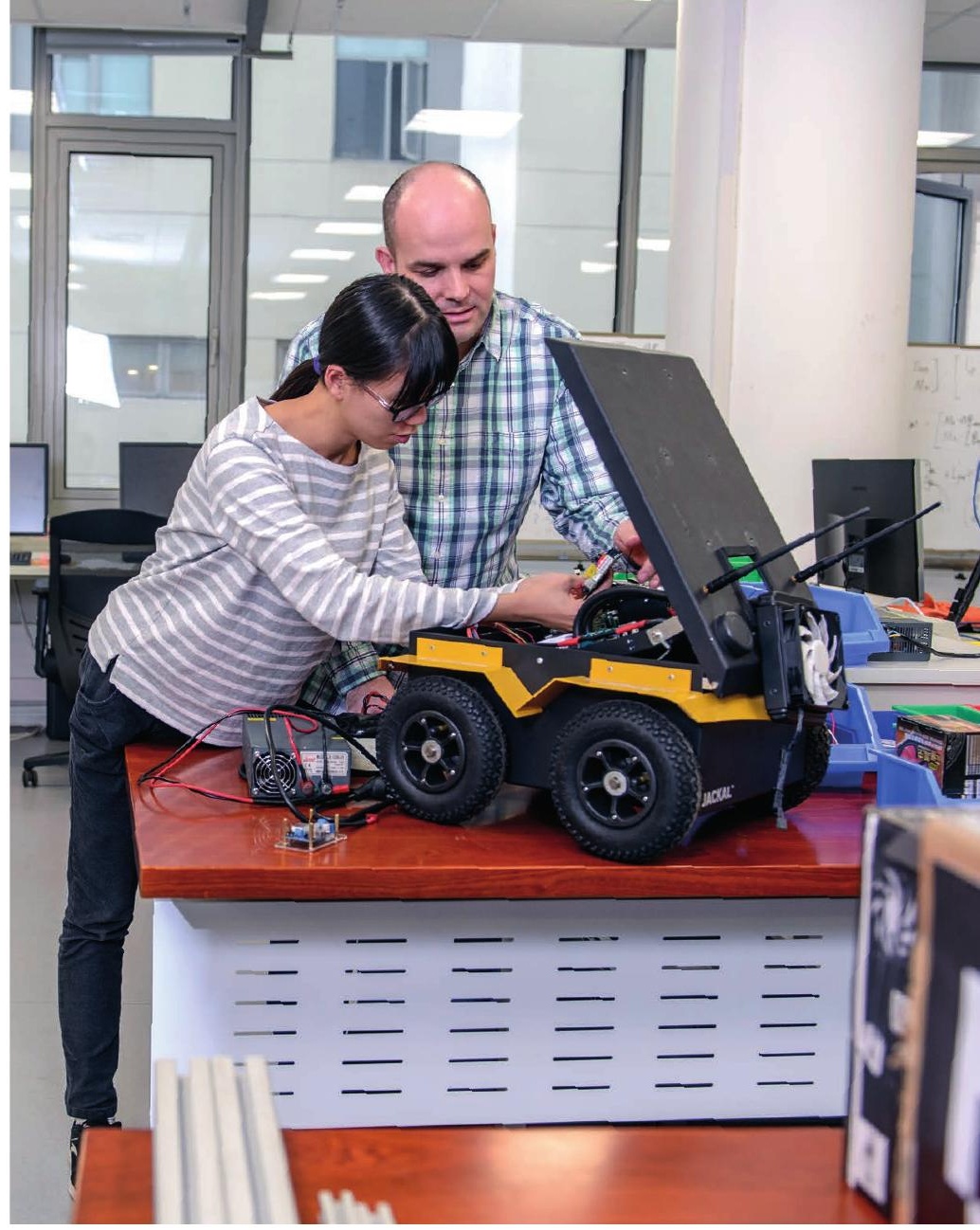

Sören Schwertfeger and Jiawei Hou work on a robot at ShanghaiTech.

Baidu's presence in the United States has also led to the adoption of a work culture more closely associated with tech start-ups in the West than the often-hierarchic nature of Chinese companies, says Wang, adding that English is the working language for both Chinese and nonChinese employees in their US centres. "We have a flat structure," he says, "and we encourage our employees to express their opinions freely, to enjoy being challenged." -

Owen Churchill is a journalist living in London who writes on tech, culture, and media in China.

\section{AI PROJECTS TO WATCH}

\section{DEEPBRAIN}

- Artificial intelligence (Al) company developing speech-recognition software focused on the Chinese market.

- Small team of around 20 now focused on cloud-based services.

- Recently received 32 million yuan (US\$4.8 million) in finance.

\section{IFLYTEK}

- Ranked sixth in MIT Technology Review's '50 Smartest Companies 2017'.

- Developed voicerecognition software used by hundreds of millions of people in China.

- Valued at 84 billion yuan on the Shenzhen Stock Exchange.

\section{CAMBRICON}

- Government-backed deep-learning processor chip.

- Received 10 million yuan in funding in 2017.

- Aims to challenge Google's AlphaGo, a program that plays the Chinese board game Go, using a computer that's a fraction of the size.

\section{BAIDU'S DEEP-LEARNING LABORATORY}

- Collaboration between Chinese government and Baidu.

- Launched in 2017 with an undisclosed budget.

- Multiple universities involved, including Beihang, which is known for military research.

\section{BAIDU SEATTLE RESEARCH CENTER}

- Baidu's latest US research and development facility, following two in Silicon Valley.

- Facility to focus on Al and cloud computing.

- Seeks to tap into the Seattle region's computing talent.

\section{TENCENT SEATTLE AI LAB}

- First US-based Al lab for Asia's most valuable company.

- The lab will employ around 20 people to work on areas such as natural language understanding.

- Lab to be headed by former Microsoft scientist Yu Dong. 